\title{
Linear quadratic optimal boundary control of a diffusion-convection-reaction system
}

\author{
L. Mohammadi, I. Aksikas, S. Dubljevic, J. F. Forbes \\ Department of Chemical and Materials Engineering \\ University of Alberta, Edmonton, Ab, T6G 2G6, Canada \\ \{leily,aksikas, stevan.dubljevic, fraser.forbes\}@ualberta.ca
}

\begin{abstract}
In this work, the boundary control of a distributed parameter system (DPS) modeled by parabolic partial differential equations with spatially varying coefficients is studied. An infinite dimensional state space setting is formulated and an exact transformation of the boundary actuation is realized to obtain an evolutionary model. The evolutionary model is used for subsequent linear quadratic regulator synthesis which incorporates the spatially varying coefficients of the underlying set of the PDEs. The formulated LQR controller is applied to the nonlinear model of the system and its performance is studied.
\end{abstract}

Keywords: Distributed parameter systems, Parabolic PDEs, LQ controller, Boundary control

\section{INTRODUCTION}

The dynamics of many unit operations in chemical industry can be described by a set of partial differential equations (PDEs). A prime example of these systems is a fixed bed reactor with axial dispersion. In order to capture effects of reactions, diffusion and convection phenomena within the reactor, the reactor model is given by a set of parabolic partial differential equations. In recent years, research on control of DPS has focused on methodologies that deal with infinite dimensional nature of these systems [Curtain and Zwart (1995); Bensoussan et al. (1992)]. In the aforementioned works, distributed parameter systems are formulated in a state space form similar to lumped parameter systems by introducing a suitable infinite dimensional space setting and associated operators. Subsequently, the infinite dimensional controllers are formulated for the infinite dimensional space realization of the system [Curtain and Zwart (1995), Callier and Winkin (1990), Callier and Winkin (1992)].

Within the class of distributed parameter systems, Aksikas et al.(2008, 2009) studied the solution of LQ control problem for hyperbolic systems by solving an operator Riccati equation. Under some assumptions, the operator Riccati equation can be converted to an equivalent Matrix Riccati equation, which can be solved numerically. However, this algorithm cannot be used for the parabolic systems, as the operator Riccati equation cannot be converted to a Matrix Riccati equation for such systems.

For parabolic PDEs Christofides (2001) studied nonlinear order reduction and control of nonlinear parabolic systems. For diffusion-reaction systems, which are described by parabolic PDEs, Dubljevic et al. (2006) used modal decomposition to derive finite-dimensional systems that capture the dominant dynamics of the original PDE and are subsequently used for the low dimensional predictive controller design.

In this work we are interested in the boundary control of a system described by a set of nonlinear parabolic PDEs.
Linearizing the nonlinear equations about the steady state profile of the system results in a set of linear parabolic PDEs with spatially varying coefficients. The control problem is transformed into a well posed abstract boundary control problem by applying an exact transformation. The spectrum of the resulting linear operator is computed by adopting the solution method of the heat equation for composite media to solve the eigenvalue problem. Finally, by using the system spectral properties, the operator Riccati equation is converted to a set of coupled algebraic equations which are solved numerically.

As a case study, we consider a tubular reactor wherein the Van de Vusse reaction takes place. This reaction scheme consists of two series parallel reactions. The material balance for the reactor results in a set of coupled nonlinear parabolic PDEs, in particular a triangular operator. The triangular structure simplifies the computation of the spectrum of the system. Triangularization of a general parabolic operator is the subject of our future work.

\section{MODEL DESCRIPTION}

The chemical process considered in this work is a tubular reactor with axial dispersion and involves the following series parallel reactions (Van de Vusse reaction):

$$
\begin{aligned}
& A \stackrel{k_{1}}{\longrightarrow} B \stackrel{k_{2}}{\longrightarrow} C \\
& A+A \stackrel{k_{3}}{\longrightarrow} D
\end{aligned}
$$

where the rate equations are:

$$
r_{A}=-k_{1} C_{A}-k_{3} C_{A}^{2}, \quad r_{B}=k_{1} C_{A}-k_{2} C_{B}
$$

To model the reactor, an isothermal process is considered. Therefore the dynamics of the system are described by the following mass balance parabolic partial differential equations (PDE's):

$$
\begin{aligned}
& \frac{\partial C_{A}}{\partial t}=D_{a} \frac{\partial^{2} C_{A}}{\partial z^{2}}-v \frac{\partial C_{A}}{\partial z}+r_{A}, \\
& \frac{\partial C_{B}}{\partial t}=D_{a} \frac{\partial^{2} C_{B}}{\partial z^{2}}-v \frac{\partial C_{B}}{\partial z}+r_{B}
\end{aligned}
$$


with associated initial and boundary conditions:

$$
\begin{aligned}
& \left.D_{a} \frac{\partial C_{A}}{\partial z}\right|_{z=0}=v\left(\left.C_{A}\right|_{z=0}-C_{A_{i n}}\right) \\
& \left.D_{a} \frac{\partial C_{B}}{\partial z}\right|_{z=0}=v\left(\left.C_{B}\right|_{z=0}-C_{B_{i n}}\right) \\
& \left.\frac{\partial C_{A}}{\partial z}\right|_{z=l}=0,\left.\quad \frac{\partial C_{B}}{\partial z}\right|_{z=l}=0 \\
& C_{A}(z, 0)=C_{A_{0}}(z), \quad C_{B}(z, 0)=C_{B_{0}}(z)
\end{aligned}
$$

In the Eqs. 3-4, $C_{A}, C_{B}, D_{a}, v, C_{A_{i n}}$, and $C_{B_{i n}}$ denote the concentrations of reactant $\mathrm{A}$ and $\mathrm{B}$, the axial dispersion coefficient, the superficial velocity, the inlet concentration of component $\mathrm{A}$, and the inlet concentration of component $\mathrm{B}$, respectively.

The corresponding steady-state equations of the PDE model given by Eq. 3 are given as the solution of the following ordinary differential equations:

$$
\begin{aligned}
& D_{a} \frac{\partial^{2} C_{A_{s s}}}{\partial z^{2}}-v \frac{\partial C_{A_{s s}}}{\partial z}-k_{1} C_{A_{s s}}-k_{3} C_{A_{s s}}^{2}=0 \\
& D_{a} \frac{\partial^{2} C_{B_{s s}}}{\partial z^{2}}-v \frac{\partial C_{B_{s s}}}{\partial z}+k_{1} C_{A_{s s}}-k_{2} C_{B_{s s}}=0
\end{aligned}
$$

\subsection{Linearized model}

Let us define the following state variables:

$$
\theta(t)=\left[\begin{array}{l}
\theta_{1} \\
\theta_{2}
\end{array}\right]=\left[\begin{array}{l}
C_{A}-C_{A_{s s}} \\
C_{B}-C_{B_{s s}}
\end{array}\right]
$$

and let us assume that the inlet concentration of $A, C_{A_{i n}}$ is the manipulated variable, and the new input is:

$$
u(t)=v\left(C_{A_{i n}}-C_{A_{\text {in,ss }}}\right)
$$

Then the linearization of the system defined by Eq. 3 around its steady state profile leads to the following linear infinite dimensional system:

$$
\frac{\partial \theta}{\partial t}=\left[\begin{array}{cc}
D_{a} \frac{\partial^{2}}{\partial z^{2}}-v \frac{\partial}{\partial z}-\tilde{k}_{1} & 0 \\
k_{1} & D_{a} \frac{\partial^{2}}{\partial z^{2}}-v \frac{\partial}{\partial z}-k_{2}
\end{array}\right] \theta
$$

where $\tilde{k_{1}}=k_{1}+2 k_{3} C_{A_{s s}}(z)$, with the following initial and boundary conditions:

$$
\begin{aligned}
& D_{a} \frac{\partial \theta(0, t)}{\partial z}=v \theta(0, t)-\left[\begin{array}{l}
u \\
0
\end{array}\right] \\
& D_{a} \frac{\partial \theta(l, t)}{\partial z}=\left[\begin{array}{l}
0 \\
0
\end{array}\right] \\
& \theta(z, 0)=\theta_{0}=\left[\begin{array}{l}
\theta_{1_{0}}(z) \\
\theta_{2_{0}}(z)
\end{array}\right]
\end{aligned}
$$

where $\theta_{1_{0}}=C_{A_{0}}-C_{A_{s s}}$ and $\theta_{2_{0}}=C_{B_{0}}-C_{B_{s s}}$.

The linearized model given by Eq. 8 is a set of linear Sturm-Liouville equations and can be converted into a diffusion-reaction system by applying the following transformation

$$
x=\left[\begin{array}{l}
x_{1} \\
x_{2}
\end{array}\right]=\left[\begin{array}{l}
e^{-\frac{v}{2 D} z} \theta_{1} \\
e^{-\frac{v}{2 D} z} \theta_{2}
\end{array}\right]
$$

The resulting equations are:

$$
\frac{\partial}{\partial t}\left[\begin{array}{l}
x_{1} \\
x_{2}
\end{array}\right]=\left[\begin{array}{cc}
D_{a} \frac{\partial^{2}}{\partial z^{2}}-\hat{k}_{1} & 0 \\
\hat{k_{2}} & D_{a} \frac{\partial^{2}}{\partial z^{2}}-\hat{k}_{3}
\end{array}\right]\left[\begin{array}{l}
x_{1} \\
x_{2}
\end{array}\right]
$$

with the following boundary and initial conditions:

$$
\begin{aligned}
& D_{a} \frac{\partial}{\partial z}\left[\begin{array}{l}
x_{1} \\
x_{2}
\end{array}\right]_{z=0}=\frac{v}{2}\left[\begin{array}{l}
x_{1} \\
x_{2}
\end{array}\right]_{z=0}-\left[\begin{array}{l}
u \\
0
\end{array}\right] \\
& D_{a} \frac{\partial}{\partial z}\left[\begin{array}{l}
x_{1} \\
x_{2}
\end{array}\right]_{z=l}=-\frac{v}{2}\left[\begin{array}{l}
x_{1} \\
x_{2}
\end{array}\right]_{z=l} \\
& x(z, 0)=x_{0}
\end{aligned}
$$

where $x_{0}=e^{-\frac{v}{2 D_{a}} z} \theta_{0}$ and $\hat{k_{1}}=-\frac{v^{2}}{4 D_{a}}-k_{1}-2 k_{3} C_{A_{s s}}(z)$, $\hat{k_{2}}=k_{1}, \hat{k_{3}}=-\frac{v^{2}}{4 D_{a}}-k_{2}$.

\subsection{Infinite-Dimensional representation}

The system given by Eqs. 10-11 can be formulated as an abstract boundary control problem on the Hilbert space $H:=L^{2}(0, l) \times L^{2}(0, l)$ [Curtain and Zwart (1995)],

$$
\begin{aligned}
& \frac{d x(t)}{d t}=\mathfrak{A} x(t), \quad x(0)=x_{0} \\
& \mathfrak{B} x(t)=u(t) \\
& y(t)=\mathfrak{C} x(t)
\end{aligned}
$$

The operator $\mathfrak{A}$ is given as follows:

$\mathfrak{A}=\left[\begin{array}{cc}D_{a} \frac{d^{2}}{d z^{2}}-\hat{k}_{1}(z) & 0 \\ \hat{k}_{2} & D_{a} \frac{d^{2}}{d z^{2}}-\hat{k_{3}}\end{array}\right]:=\left[\begin{array}{cc}A_{11} & 0 \\ A_{21} & A_{22}\end{array}\right]$

with the domain:

$$
\begin{aligned}
\mathcal{D}(\mathfrak{A})=\{x \in H: x & \text { is a.c. }, \frac{d x}{d z} \in H \text { and } \\
D_{a} \frac{\partial x_{1}(l, t)}{\partial z} & =-\frac{v}{2} x_{1}(l, t), D_{a} \frac{\partial x_{2}(0, t)}{\partial z}=\frac{v}{2} x_{2}(0, t), \\
D_{a} \frac{\partial x_{2}(l, t)}{\partial z} & \left.=-\frac{v}{2} x_{2}(l, t)\right\}
\end{aligned}
$$

The boundary operator $\mathfrak{B}: H \rightarrow R$ is given as:

$$
\mathfrak{B} x(z)=\left[\begin{array}{ll}
D_{a} \frac{d}{d z}(0)-v & 0
\end{array}\right]\left[\begin{array}{l}
x_{1}(0) \\
x_{2}(0)
\end{array}\right]
$$

with the domain:

$$
\mathcal{D}(\mathfrak{B})=\left\{x \in H: x \text { is a.c. }, \frac{d x}{d z} \in H\right\}
$$

and the output operator, $\mathfrak{C}$, is defined by the available measurement and is given by:

$$
y(t):=\mathfrak{C} x(\cdot)=\int_{0}^{l} \delta\left(z-z_{c}\right) x(\cdot) d z
$$

where $\delta$ is approximated Dirac function and the measurement is applied at $z_{c}$.

A new operator $\mathcal{A}$ is defined by:

$$
\mathcal{A} x=\mathfrak{A} x
$$

$$
\begin{array}{r}
\mathcal{D}(\mathcal{A})=\mathcal{D}(\mathfrak{A}) \cap \operatorname{ker}(\mathfrak{B})=\left\{x \in H: x \text { is a.c. }, \frac{d x}{d z} \in H\right. \text { and } \\
\left.D_{a} \frac{\partial x(0, t)}{\partial z}+\frac{v}{2} x(0, t)=0, D_{a} \frac{\partial x(l, t)}{\partial z}+\frac{v}{2} x(l, t)=0\right\}
\end{array}
$$

Let us assume that $\mathcal{A}$ is the infinitesimal generator of a $C_{0}$-semigroup on $H$ and there exist a $B$ such that for all $u, B u \in \mathcal{D}(\mathfrak{A})$, and the following holds:

$$
\mathfrak{B} B u=u, \quad u \in U:=R
$$


Function $B$ is chosen to be $B=\left[\begin{array}{l}B_{1} \\ B_{2}\end{array}\right]$ where

$$
\begin{aligned}
& B_{1}=\frac{1}{4 D_{a} l+v l^{2}} z^{2}-\frac{1}{v} \\
& B_{2}=-\frac{2}{4 D_{a} l+v l^{2}} z^{2}+\frac{1}{4 D_{a}+v l} z+\frac{4 D_{a}}{4 D_{a} v+v^{2} l}
\end{aligned}
$$

By defining a new input $\tilde{u}(t)=\dot{u}(t)$ and a new state $x^{e}(t)=[u(t) x(t)-B u(t)]^{\prime}$, the problem can be reformulated on the extended state space $H^{e}=H \oplus U$ as:

$$
\begin{aligned}
& \dot{x}^{e}(t)=A^{e} x^{e}(t)+B^{e} \tilde{u}(t) \\
& y=C^{e} x^{e}
\end{aligned}
$$

where

$$
A^{e}=\left[\begin{array}{cc}
0 & 0 \\
\mathfrak{A} B & \mathcal{A}
\end{array}\right], \quad B^{e}=\left[\begin{array}{c}
I \\
-B
\end{array}\right], \quad C^{e}=\mathfrak{C}\left[\begin{array}{ll}
B & I
\end{array}\right]
$$

\section{EIGENVALUE PROBLEM}

\subsection{Eigenvalues and eigenfunctions of $A_{11}$ and $A_{22}$}

The operators $A_{11}$ and $A_{22}$ given by Eq.13 are both selfadjoint Sturm-Liouville operators. Since $A_{22}$ is a linear operator with constant coefficients, calculation of the spectrum $\sigma\left(A_{22}\right)$ is straightforward and eigenvalues are given by

$$
\mu_{n}^{2}=D_{a} \omega_{n}^{2}+\hat{k}_{3}
$$

where $\omega_{n}$ is the solution to the following equation

$$
\tan (\omega l)=\frac{4 D_{a} \omega v}{4 D_{a}^{2} \omega^{2}-v^{2}}
$$

and the corresponding eigenfunctions are given by

$$
\psi_{n}=\cos \left(\omega_{n} z\right)+\frac{v}{2 D_{a} \omega_{n}} \sin \left(\omega_{n} z\right)
$$

However, due to linearization around the steady state trajectory, the linearized reaction coefficients for $A_{11}$ depend on $z$. Therefore, the calculation of the spectrum of the operator $A_{11}$ is a challenging issue. In this work, the solution method of the heat equation for composite media is adopted to solve the eigenvalue problem for Sturm-Liouville operator with spatially varying coefficients. Namely, the length of the reactor is divided to a finite number of sections. It is assumed that at each layer the values of coefficients are constant. The mathematical formulation of this approach can be given as [de. Monte (2002)]:

$\frac{\partial \vartheta_{i}}{\partial t}=D \frac{\partial^{2} \vartheta_{i}}{\partial^{2} z}+k_{i} \vartheta_{i}, \quad \vartheta_{i}=x_{1}(z), z \in\left[z_{i-1}, z_{i}\right], i=1, \cdots, N$

Boundary condition at $z=0$ :

$$
D \frac{\partial \vartheta_{1}(0, t)}{\partial z}=h_{1} \vartheta_{1}(0, t), \quad h_{1}=\frac{v}{2}
$$

Continuity boundary conditions:

$$
\begin{aligned}
\vartheta_{i-1}\left(z_{i}, t\right) & =\vartheta_{i}\left(z_{i}, t\right) \quad(i=2,3, \cdots, N) \\
\left(\frac{\partial \vartheta_{i-1}}{\partial z}\right)_{x_{i}} & =\left(\frac{\partial \vartheta_{i}}{\partial z}\right)_{x_{i}} \quad(i=2,3, \cdots, N)
\end{aligned}
$$

Boundary condition at $x=L$

$$
D \frac{\partial \vartheta_{N}(L, t)}{\partial z}=-h_{N} \vartheta_{N}(L, t), \quad h_{N}=\frac{v}{2}
$$

The continuity boundary conditions imply that at the interfaces of the layers the temperature and the flux are the same in two adjacent layers. The PDEs should be solved for each layer with unspecified boundary data at the interfaces. Then the solutions can be determined by applying the matching conditions.

The Eqs.26-30 are solved by the method of separation of variables. We will have:

$$
\vartheta_{i}(z, t)=Z_{i}(z) G_{i}(t), \quad z \in\left[z_{i}, z_{i+1}\right]
$$

Substituting the above equation in the original PDE, we obtain:

$$
D \frac{\partial^{2} Z_{i}}{\partial^{2} z}+k_{i} Z_{i}=-\lambda_{i}^{2} Z_{i}
$$

which can be written as:

$$
D \frac{\partial^{2} Z_{i}}{\partial^{2} z}+\varpi_{i}^{2} Z_{i}=0, \quad \varpi_{i}^{2}=k_{i}+\lambda_{i}^{2} ;
$$

Eigenfunctions associated with Eq.33 have the form of:

$$
Z_{i}(z)=a_{i} \sin \left(\varpi_{i} x\right)+b_{i} \cos \left(\varpi_{i} x\right)
$$

$a_{i}$ and $b_{i}$ are integration constants and should be calculated by the boundary conditions at each layer. Since the PDEs are coupled through boundary conditions these coefficients are calculated simultaneously. Finally $Z_{i}$ can be obtained as:

$Z_{i}(z)=a_{1} \Phi_{i}\left(\varpi_{i}\right)\left(\sin \left(\varpi_{i} z\right)+\Gamma_{i} \cos \left(\varpi_{i} z\right)\right), z \in\left[z_{i}, z_{i+1}\right]$

where

$$
\Phi_{1}=1 ; \quad \Phi_{i}\left(\varpi_{1}, \cdots, \varpi_{i}\right)=\Phi_{i, i-1} \Phi_{i-1, i-2} \cdots \Phi_{2,1}
$$

and

$$
\begin{gathered}
\Phi_{i, i-1}=\frac{\left(\sin \left(\varpi_{i-1} z_{i}\right)+\Gamma_{i-1} \cos \left(\varpi_{i-1} z_{i}\right)\right)}{\left(\sin \left(\varpi_{i} z_{i}\right)+\Gamma_{i} \cos \left(\varpi_{i} z_{i}\right)\right)} \\
\Phi_{M, M-1}=\frac{1}{k_{M}} \frac{\left(\cos \left(\varpi_{M-1} z_{i}\right)-\Gamma_{M-1} \sin \left(\varpi_{M-1} z_{M}\right)\right)}{\left(\cos \left(\varpi_{M} z_{M}\right)-\Gamma_{M} \sin \left(\varpi_{M} z_{M}\right)\right)} \\
\Gamma_{1}=-\frac{h_{1} \sin \left(\varpi_{1} z_{1}\right)-k_{1} \varpi_{1} \cos \left(\varpi_{1} z_{1}\right)}{h_{1} \cos \left(\varpi_{1} z_{1}\right)+k_{1} \varpi_{1} \sin \left(\varpi_{1} z_{1}\right)} \\
\Gamma_{i}=\frac{\cos \left(\varpi_{i} z_{i}\right) \xi_{i}-\sin \left(\varpi_{i} z_{i}\right) \zeta_{i}}{\sin \left(\varpi_{i} z_{i}\right) \xi_{i}+\cos \left(\varpi_{i} z_{i}\right) \zeta_{i}} \\
\Gamma_{M}=-\frac{h_{M+1} \sin \left(\varpi_{M} z_{M+1}\right)+k_{M} \varpi_{M} \cos \left(\varpi_{M} z_{M+1}\right)}{h_{M+1} \cos \left(\varpi_{M} z_{M+1}\right)-k_{M} \varpi_{i} \sin \left(\varpi_{M} z_{M+1}\right)}
\end{gathered}
$$

where

$$
\begin{aligned}
& \xi_{i}=\sin \left(\varpi_{i-1} z_{i}\right)+\Gamma_{i-1} \cos \left(\varpi_{i-1} z_{i}\right) \\
& \zeta_{i}=\cos \left(\varpi_{i-1} z_{i}\right)-\Gamma_{i-1} \sin \left(\varpi_{i-1} z_{i}\right)
\end{aligned}
$$

$\Gamma_{M}$ is evaluated by both Eq.38b for $i=M$ and Eq.38c [de. Monte (2002)]. Therefore one can compute $\lambda$ by comparing these equations. Solution is obtained by using numerical methods. There are infinite number of eigenvalues satisfying this condition and each has a corresponding eigenfunction of the form of Eq.35.

\subsection{Eigenvalues and eigenfunctions of the operator $\mathcal{A}$}

Let $\lambda_{n}$ and $\chi_{n}$ be eigenvalues and eigenfunctions of operator $A_{11}$ and $\mu_{n}$ and $\psi_{n}$ be eigenvalues and eigenfunctions of the operator $A_{22}$ computed by the method described in section 3.1. 
Since the operator $\mathcal{A}$ is triangular, its eigenvalues consist of eigenvalues of $A_{11}$ and $A_{22} ; \sigma(\mathcal{A})=\sigma\left(A_{11}\right) \cup \sigma\left(A_{22}\right)$ [Winkin et al. (2000)] and are given by:

$$
\begin{aligned}
\sigma_{2 n+1}=\lambda_{n}, & \text { for } n \geq 0 \\
\sigma_{2 n}=\mu_{n}, & \text { for } n \geq 1
\end{aligned}
$$

with corresponding eigenvectors given by

$$
\begin{aligned}
\phi_{2 n+1} & =\left[\begin{array}{c}
\chi_{n} \\
\left(\lambda_{n} I-A_{22}\right)^{-1} A_{21} \chi_{n}
\end{array}\right] \\
\phi_{2 n} & =\left[\begin{array}{c}
0 \\
\psi_{n}
\end{array}\right]
\end{aligned}
$$

and the corresponding biorthonormal eigenfunctions are:

$$
\begin{aligned}
\Psi_{2 n+1} & =\left[\begin{array}{c}
\chi_{n} \\
0
\end{array}\right] \\
\Psi_{2 n} & =\left[\begin{array}{c}
\left(\mu_{n} I-A_{11}\right)^{-1} A_{21} \psi_{n} \\
\psi_{n}
\end{array}\right]
\end{aligned}
$$

where

$$
\begin{aligned}
\left(\mu_{n} I-A_{11}\right)^{-1} A_{21} \psi_{n} & =\sum_{n=0}^{\infty} \frac{1}{\mu_{n}-\lambda_{m}}\left\langle A_{21} \psi_{n}, \chi_{m}\right\rangle \chi_{m} \\
\left(\lambda_{n} I-A_{22}\right)^{-1} A_{21} \chi_{n} & =\sum_{n=0}^{\infty} \frac{1}{\lambda_{n}-\mu_{m}}\left\langle A_{21} \chi_{n}, \psi_{m}\right\rangle \psi_{m}
\end{aligned}
$$

\subsection{Eigenvalues and eigenfunctions of the operator $A^{e}$}

It can be demonstrated that the $\sigma\left(A^{e}\right)=\sigma(A) \cup\{0\}$, and the corresponding eigenfunctions on the extended space is given by:

$$
\hat{\phi}_{0}=\left[\begin{array}{c}
1 \\
-\mathcal{A}^{-1}(\mathfrak{A} B)
\end{array}\right]=\left[\begin{array}{c}
1 \\
\sum_{m=0}^{\infty} \frac{1}{\sigma_{m}}\left\langle\mathfrak{A} B, \Psi_{m}\right\rangle \phi_{m}
\end{array}\right]
$$

and

$$
\hat{\phi}_{n}=\left[\begin{array}{c}
0 \\
\phi_{n}
\end{array}\right], \quad n \geq 1
$$

where $\phi_{n}$ is the eigenfunction of the operator $\mathcal{A}$. Calculation of the associated biorthonormal eigenfunctions is straightforward and given by:

$$
\begin{aligned}
& \hat{\Psi}_{0}=\left[\begin{array}{l}
1 \\
0
\end{array}\right] \\
& \hat{\Psi}_{n}=\left[\begin{array}{c}
\frac{1}{\sigma_{n}}(\mathfrak{A} B)^{*} \Psi_{n} \\
\Psi_{n}
\end{array}\right]
\end{aligned}
$$

where $\sigma_{n}$ and $\Psi_{n}$ are the eigenvalues and the corresponding eigenfunctions of $A^{*}$.

\section{TRAJECTORY AND STABILITY ANALYSIS}

Operator $\mathcal{A}$ has a lower triangular form and its diagonal elements are Sturm-Liouville operators. Eigenvalues of a Sturm-Liouville problem are real, countable and simple. Moreover, $A_{11}, A_{22}$ are the infinitesimal generators of the $C_{0}$-semigroups $T_{11}$ and $T_{22}$ respectively [Winkin et al. (2000); Delattre et al. (2003)]. As it is mentioned in 3.2 , eigenvalues of $\mathcal{A}$ consist of eigenvalues of $A_{11}$ and $A_{22}$. Therefore the operator $\mathcal{A}$ has real, countable and simple eigenvalues. Moreover, eigenfunctions of $\mathcal{A}$ and its adjoint are biorthogonal. Thus the operator $\mathcal{A}$ is a Riesz spectral operator [Delattre et al. (2003)]. By Curtain and
Zwart (1995), Lemma 3.2.2, operator $\mathcal{A}$ is the infinitesimal generator of the $C_{0}$-semigroup $\mathrm{T}$ given by

$$
T(t)=\left[\begin{array}{cc}
T_{11}(t) & 0 \\
T_{21}(t) & T_{22}(t)
\end{array}\right]
$$

where

$$
T_{21}(t) x_{1}=\int_{0}^{t} T_{22}(t-s) A_{21} T_{11}(s) x_{1} d s
$$

By the same approach one can deduce that the operator $A^{e}$ is a Riesz spectral operator and generates a $C_{0^{-}}$- semigroup given by:

$$
T^{e}(t)=\left[\begin{array}{cc}
I & 0 \\
S(t) & T(t)
\end{array}\right]
$$

where $S(t) x=\int_{0}^{t} T(s) \mathfrak{A} B x d s$, and $T(t)$ is the $C_{0^{-}}$ semigroup generated by $\mathcal{A}$.

By Curtain and Zwart (1995), theorem 5.2.10, necassary and sufficient conditions for a Riesz-spectral system $\sum(A, B, C)$ to be $\beta$-exponentially stabilizable are that there exist an $\epsilon>0$ such that $\sigma_{\beta-\epsilon}^{+}(A)$ comprises, at most, finitely many eigenvalues and

$$
\operatorname{rank}\left(\left\langle b, \psi_{n}\right\rangle\right)=1
$$

for all $\mathrm{n}$ such that $\lambda_{n} \in \sigma_{\beta-\epsilon}^{+}(A)$.

The spectrum of a Surm-Liouville system is bounded from above by a number [Delattre et al. (2003)], therefore the first condition of the above mentioned theorem holds for this system. It can be demonstrated that the second condition holds for our case study.

\section{OPTIMAL CONTROL DESIGN}

The linear quadratic control problem on an infinite-time horizon employs the cost function:

$$
J\left(x_{0}, u\right)=\int_{0}^{\infty}\langle y(s), y(s)\rangle+\langle u(s), R u(s)\rangle d s
$$

where $u(s)$ and $y(s)$ are the input and output trajectories, respectively, and $\mathrm{R}$ is a self-adjoint, coercive operator in $\mathcal{L}(U)$. The output function $y(\cdot)$ is given by Eq.21.

The solution of this optimal control problem can be obtained by solving the following algebraic Riccati equation (ARE):

$$
\begin{aligned}
\left\langle A^{e} x_{1}, \Pi x_{2}\right\rangle+\left\langle\Pi x_{1}, A^{e} x_{2}\right\rangle & +\left\langle C^{e} x_{1}, C^{e} x_{2}\right\rangle \\
& -\left\langle B^{e^{*}} \Pi x_{1}, R^{-1} B^{e^{*}} \Pi x_{2}\right\rangle=0
\end{aligned}
$$

When $\left(A^{e}, B^{e}\right)$ is exponentially stabilizable and $\left(C^{e}, A^{e}\right)$ is exponentially detectable, the algebraic Riccati equation 50 has a unique nonnegative self-adjoint solution $\Pi \in \mathcal{L}(\mathcal{H})$ and for any initial state $x_{0} \in \mathcal{H}$ the quadratic cost 49 is minimized by the unique control $u_{\text {opt }}$ given by

$$
u_{o p t}\left(t ; x_{0}\right)=-R^{-1} B^{e^{*}} \Pi x_{o p t}(t)
$$

In addition, the optimal cost is given by $J\left(x_{0}, u_{o p t}\right)=$ $\left\langle x_{0}, \Pi x_{0}\right\rangle$.

If we take $z_{1}=\hat{\phi}_{m}$ and $z_{2}=\hat{\phi}_{n}$, then the Riccati equation 50 becomes: 


$$
\begin{aligned}
\left\langle A \hat{\phi}_{n}, \Pi \hat{\phi}_{m}\right\rangle+ & \left\langle\Pi \hat{\phi}_{n}, A \phi_{m}\right\rangle+\left\langle C \hat{\phi}_{n}, C \hat{\phi}_{m}\right\rangle \\
& -\left\langle B^{*} \Pi \hat{\phi}_{n}, R^{-1} B^{*} \Pi \hat{\phi}_{m}\right\rangle=0
\end{aligned}
$$

If we assume that the solution has the form $\Pi z=$ $\sum_{n, m} \Pi_{n m}\left\langle z, \hat{\psi}_{m}\right\rangle \hat{\psi}_{n}$ where $\Pi_{n m}=\left\langle\hat{\phi}_{n}, \Pi \hat{\phi}_{m}\right\rangle$, then Eq. 52 becomes the system of infinite number of coupled scalar equations

$$
\left(\sigma_{n}+\sigma_{m}\right) \Pi_{n m}+\mathbf{C}_{n m}-\sum_{k=0}^{\infty} \sum_{k, l=0}^{\infty} \Pi_{n k} \Pi_{l m} \mathbf{B}_{n m}=0
$$

where $\mathbf{C}_{n m}=\left\langle C \hat{\phi}_{n}, C \hat{\phi}_{m}\right\rangle$, and $\mathbf{B}_{n m}=\left\langle R^{-1} B \hat{\psi}_{n}, B \hat{\psi}_{m}\right\rangle$.

\section{SIMULATION STUDY}

In this section the performance of the formulated controller is demonstrated. The LQ-controller discussed in the previous section is implemented on the nonlinear model of the reactor. Values of the model parameters are given in Table 1

The control objective is to drive the trajectory of $C_{B}$

Table 1. Model Parameters

\begin{tabular}{ccc}
\hline Parameter & Value & Unit \\
\hline$k_{1}$ & $1.39 \times 10^{-2}$ & $\mathrm{sec}^{-1}$ \\
$k_{2}$ & $2.78 \times 10^{-2}$ & $\mathrm{sec}^{-1}$ \\
$k_{3}$ & $2.77 \times 10^{-4}$ & $\mathrm{~mol} / \mathrm{l} . \mathrm{min}$ \\
$D_{a}$ & $3.33 \times 10^{-4}$ & $\mathrm{~m}^{2} / \mathrm{s}$ \\
$v$ & $9.5 \times 10^{-3}$ & $\mathrm{~m} / \mathrm{s}$ \\
$C_{A_{i n}}$ & 10 & $\mathrm{~mol} / \mathrm{l}$ \\
$C_{B_{\text {in }}}$ & 0 & $\mathrm{~mol} / \mathrm{l}$ \\
\hline
\end{tabular}

to the desired steady state profile. Using the nominal operating conditions, and the model given in Eqs.2-3, the steady state profiles of $C_{A}$ and $C_{B}$ were computed and are shown in Fig.1. Then, the nonlinear model was linearized about the steady state profile and transformed to the selfadjoint form of Eqs.10-11.

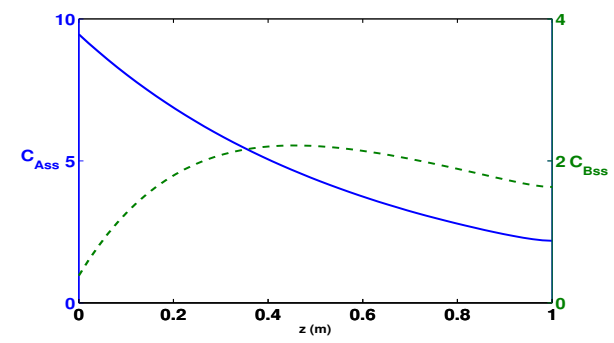

Fig. 1. Steady state profile of $C_{A}$ and $C_{B}$.

Spectrums of operators $A_{11}$ and $A_{22}$ can be calculated using the algorithm discussed in section 3.1. In order to compute the spectrum of $A_{11}$, it is assumed that the length of the reactor is divided into 50 sections and the the coefficient of the reactive term is constant in each section. First five eigenvalues of the operator $A_{11}$ are:

$$
\lambda=\{0.3080,0.3299,0.3601,0.3967,0.4380\}
$$

The spectrum of $A_{22}$ can be computed using Eqs.23-25. First five eigenvalues of $A_{22}$ are:

$$
\lambda=\{0.3136,0.3256,0.3452,0.3713,0.4031\}
$$

Finally, one can compute the spectrum of $A^{e}$ using Eqs.4339. The results are shown in Figs.2-4

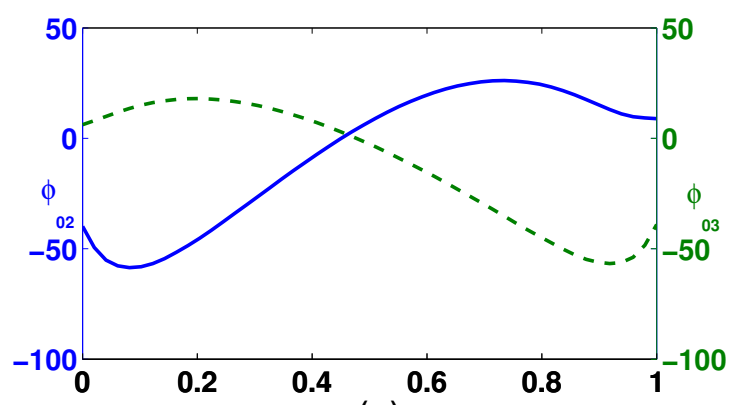

Fig. 2. Second and third elements of $\hat{\phi}_{0}$ given by Eq.43.



Fig. 3. Second element of $\hat{\phi}_{n}$ given by Eq.44 and Eqs.39-40. Note that this function is equal to zero when $n=2 k$.

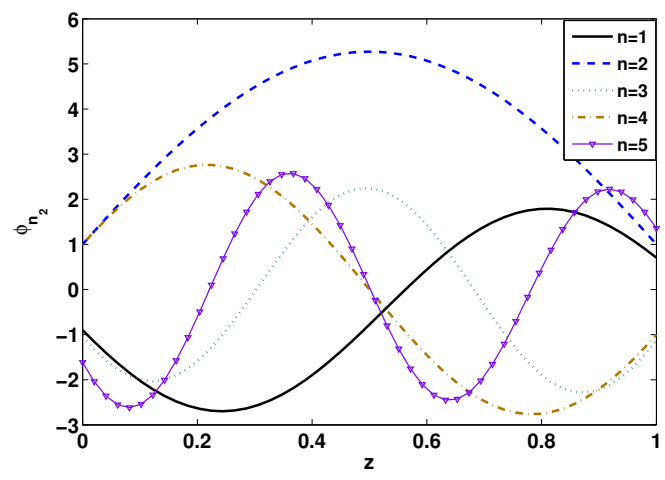

Fig. 4. Third element of $\hat{\phi}_{n}$ given by Eq.44 and Eqs.39-40.

Once the eigenvalues and eigenfunctions of the operator $A^{e}$ are calculated, the LQ-feedback controller can be computed using Eq.53. Note that since $\Pi$ is a self-adjoint operator, $\left\langle\phi_{n}, \Pi \phi_{m}\right\rangle=\left\langle\phi_{m}, \Pi \phi_{n}\right\rangle$, therefore $\Pi_{n m}=\Pi_{m n}$. As a result, Eq.53 gives $\frac{n(n+1)}{2}$ coupled algebraic equations that should be solved simultaneously where $n$ is the number of modes that are used to formulate the controller. In this work the first 21 modes are used for numerical simulation. The computed LQ controller is applied to the nonlinear model of the reactor. Simulation of the nonlinear system is done using $\mathrm{COMSOL}^{\circledR}$. The closed loop trajectory of $C_{A}$ and $C_{B}$ are shown in Figs.5-6, and the trajectory of the manipulated variable, inlet concentration of $A$, is shown in Fig.7. 


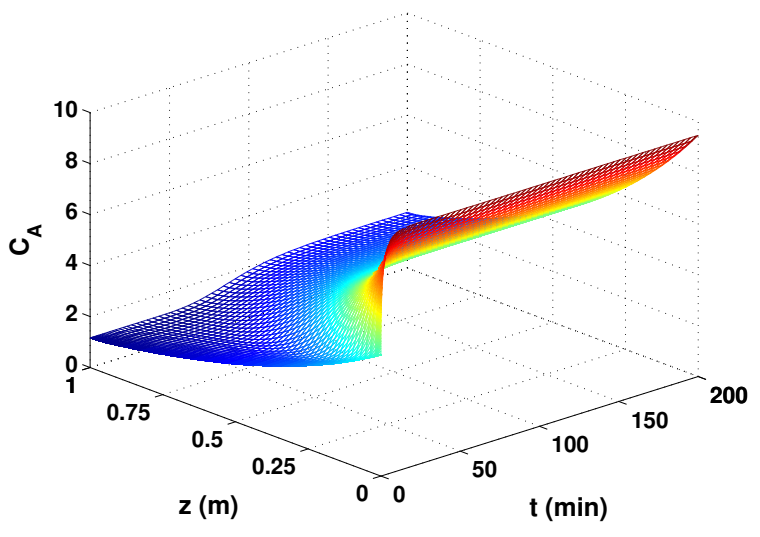

Fig. 5. Closed loop trajectory of $C_{A}$.

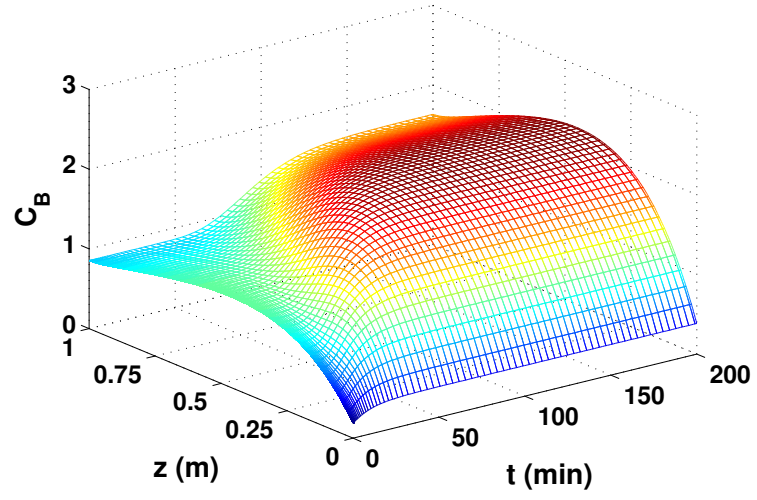

Fig. 6. Closed loop trajectory of $C_{B}$.



Fig. 7. Closed loop trajectory of the input variable (Inlet Concentration).

The tracking error is shown in Fig.8. Simulation results demonstrate that the controller is able to drive the output variable to the desired steady state profile.

\section{SUMMARY}

In this paper, optimal control of a tubular reactor with axial dispersion is studied. The reactor can be modeled by a set of nonlinear parabolic partial differential equations. Linearization around the steady state profile of the system

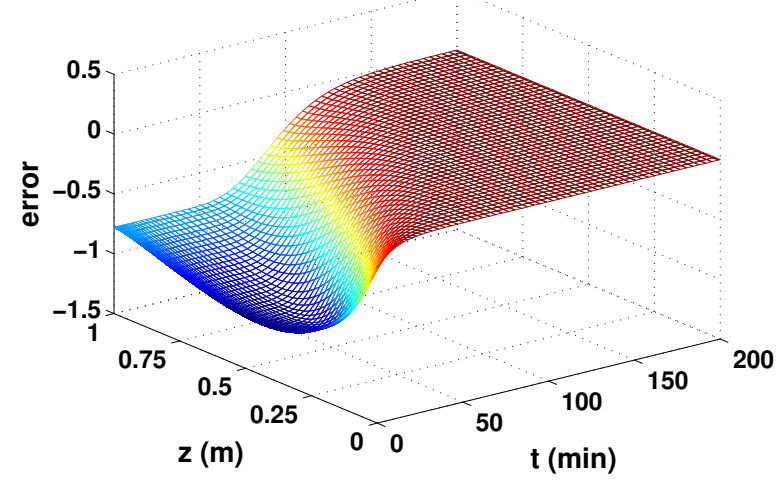

Fig. 8. Trajectory of tracking error.

results in a set of linear PDEs with spatially varying coefficients. A method similar to solution of the heat equation for composite media is used to compute the spectrum of the system. Then a LQ controller is formulated to regulate the state variables to the steady state profile.

\section{REFERENCES}

Aksikas, I., Forbes, F.J., and Belhamadia, Y. (2009). Optimal Control Design for Time-Varying Catalytic Reactors: A Riccati Equation-Bases Approach. International Journal of Control, 82(7), 1219-1228.

Aksikas, I., Winkin, J., and Dochain, D. (2008). Optimal lq-feedback control for a class of first-order hyperbolic distributed parameter systems. ESAIM: COCV , 14(4), 897-908. doi:10.1051/cocv:2008015. URL http://dx.doi.org/10.1051/cocv: 2008015.

Bensoussan, A., Delfour, M.C., Da Prato, G., and Mitter, S.K. (1992). Representation and control of infinite dimensional systems (vol. 1). Birkhauser Verlag, Basel, Switzerland, Switzerland.

Callier, F.M. and Winkin, J. (1990). Spectral factorization and lq-optimal regulation for multivariable distributed systems. Int. J. Control, 52, 55-75.

Callier, F.M. and Winkin, J. (1992). LQ-Optimal Control of Infinite-Dimensional Systems by Spectral actorization. Automatica, 28(4), 757-770.

Christofides, P.D. (2001). Nonlinear and Robust Control of PDE Systems:Methods and Applications to TransportReaction Processes. Birkhäuser.

Curtain, R.F. and Zwart, H. (1995). An introduction to infinite-dimensional linear systems theory. SpringerVerlag New York, Inc., New York, NY, USA.

de. Monte, F. (2002). An analytic approach to the unsteady heat conduction processes in one-dimensional composite media. International Journal of Heat and Mass Transfer, 45, 1333-1343.

Delattre, C., Dochain, D., and Winkin, J. (2003). Sturmliouville systems are riesz-spectral systems. Applied mathematics and computer science, 13, 481-484.

Dubljevic, S., El-Farra, N.H., Mhaskar, P., and Christofides, P.D. (2006). Predictive control of parabolic PDEs with state and control constraints. International Journal of Robust and Nonlinear Control, 16(16), 749-772.

Winkin, J.J., Dochain, D., and Ligarius, P. (2000). Dynamical analysis of distributed parameter tubular reactors. Automatica, 36(3), $349-361$. 